\title{
The Breakdown of Linear Elastic Fracture Mechanics near the Tip of a Rapid Crack
}

\author{
Ariel Livne, Eran Bouchbinder and Jay Fineberg
}

\author{
Racah Institute of Physics, Hebrew University of Jerusalem, Jerusalem 91904, Israel
}

\begin{abstract}
We present high resolution measurements of the displacement and strain fields near the tip of a dynamic (Mode I) crack. The experiments are performed on polyacrylamide gels, brittle elastomers whose fracture dynamics mirror those of typical brittle amorphous materials. Over a wide range of propagation velocities $\left(0.2-0.8 c_{s}\right)$, we compare linear elastic fracture mechanics (LEFM) to the measured near-tip fields. We find that, sufficiently near the tip, the measured stress intensity factor appears to be non-unique, the crack tip significantly deviates from its predicted parabolic form, and the strains ahead of the tip are more singular than the $r^{-1 / 2}$ divergence predicted by LEFM. These results show how LEFM breaks down as the crack tip is approached.

PACS numbers: 46.50.+a, 62.20.Mk, 89.75.Kd
\end{abstract}

The behavior of a cracked body under applied stress is of extreme practical and fundamental importance. The accepted approach to describing crack dynamics is linear elastic fracture mechanics (LEFM) [1]. This framework assumes that a material is linearly elastic with all nonlinear and dissipative processes well confined to the near tip vicinity. LEFM provides a full description of the elastic fields surrounding the tip of a single crack, whether static or propagating. Open questions, however, such as criteria for crack path selection [2], the origin of dynamic instabilities (micro-branching) [2] and oscillations of a single crack at high velocities 3] underline the need for a more detailed understanding of the near tip behavior in dynamic fracture. Studies of this region have been limited mainly to static cracks [4, [5], as detailed measurement of a microscopic region which may move at speeds approaching sound speeds entails enormous difficulties.

Here we report on recent experimental results in the dynamic fracture of brittle polyacrylamide gels. In these soft materials, fracture dynamics are identical to those observed in standard brittle amorphous materials, but crack velocities, which scale with material sound speeds, are nearly three orders of magnitude lower [6]. Thus, detailed visualization of the fields in the near-tip region within an effectively $2 \mathrm{D}$ medium becomes possible. The measurements provide a detailed description of the neartip fields. Quantitative comparison with LEFM delineates its domain of validity. These findings suggest the importance of nonlinear elasticity as the crack tip is approached.

The brittle gels used in these experiments are transparent, neo-Hookean, incompressible elastomers that were used in [3]. They are composed of $13.8 \%$ total monomer and $2.6 \%$ bis-acrylamide cross-linker concentrations. The shear $(\mu=35.2 \pm 1.4 \mathrm{kPa})$ and Young's $(E=3 \mu)$ moduli of these gels yield shear and longitudinal wave speeds of $c_{s}=5.90 \pm 0.15 \mathrm{~m} / \mathrm{s}$ and $c_{L}=11.8 \pm 0.3 \mathrm{~m} / \mathrm{s}$. Their typical dimensions were $(X \times Y \times Z) 130 \times 125 \times 0.2 \mathrm{~mm}$ where $X$, $Y$, and $Z$ are, respectively, the propagation, loading, and gel thickness directions. Reducing the gel thickness sup- presses micro-branching and enables single-crack states to attain high velocities in effectively 2D media [3].

The gels were cast between two flat glass plates. The face of one plate was randomly scratched with No. 600 Alundum lapping powder. These scratches, of $16 \mu \mathrm{m}$ mean depth, were imprinted on one of the gel faces. The resulting scratch pattern was used as a "tracer field" for visualization of the displacement field, and did not affect the crack dynamics.

Experiments were performed as in [ 6 ] by imposing uniaxial (Mode I) loading via constant displacement in the vertical $(Y)$ direction. Once the desired stress was reached, a seed crack was imposed at the sample's edge, midway between the vertical boundaries. As Fig. 1(a) shows, the displacement field of the dynamic crack that emerged was visualized at the center of the gel by a highspeed $(485 \mathrm{~Hz}$ frame rate) camera that was focused on a fixed $15.4 \mathrm{~mm}(X)$ by $12.3 \mathrm{~mm}(Y)$ region. The camera's $1280 \times 1024$ pixel resolution enabled a $12 \mu \mathrm{m}$ spatial resolution. Image blur was limited to less than a single pixel by using a $2.8 \mu$ s exposure time.

A fundamental quantity of fracture mechanics is the displacement field $\boldsymbol{u}$, defined by $\boldsymbol{x}^{\prime}=\boldsymbol{x}+\boldsymbol{u}(\boldsymbol{x})$, where $\boldsymbol{x}$ is an un-deformed 'rest' configuration and $\boldsymbol{x}^{\prime}$ a deformed one. We measured this quantity as follows. We first visualized the scratch pattern by shadowgraphy [7]. We then found the displacement field between a photograph taken immediately preceding fracture initiation (reference frame), and photographs capturing traveling cracks. This was done by means of a particle tracking technique where the scratches were tracked by crosscorrelating small boxes (10-20 pixels in length, at 5 pixel intervals) from the reference frame with corresponding regions deformed by the crack's passage. The maximum correlation for each box provided a sub-pixel measurement of the displacement field generated by the crack. Correcting for the uniform stretch of the reference frame yielded $\boldsymbol{u}(\boldsymbol{x})$ in the rest frame, thereby enabling a direct comparison to LEFM.

Let us first consider the crack tip opening displacement 


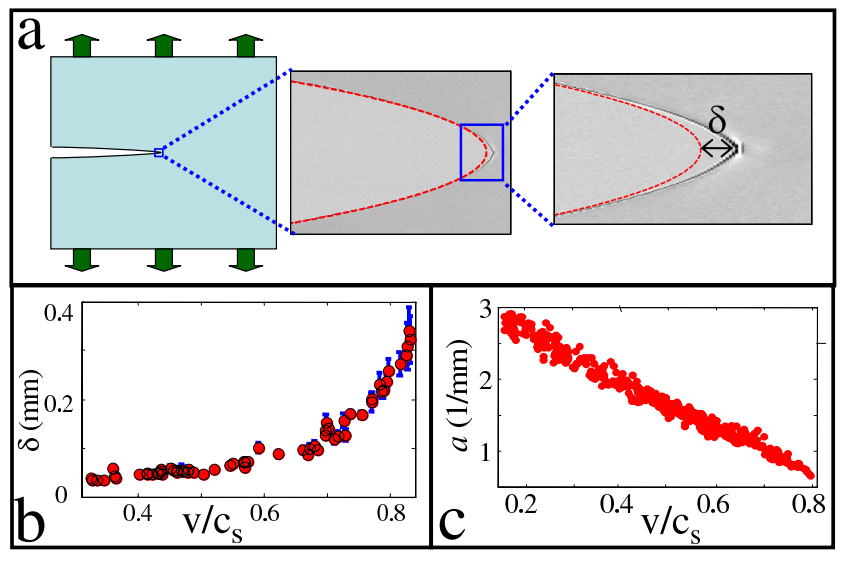

FIG. 1: (Color online) (a) (left) A single crack traveling from left to right in a (to scale) uniaxially loaded sample. (center) Photograph (here $5.4 \times 5.2 \mathrm{~mm}$ ) of the profile at the tip of a dynamic crack $\left(v \sim 0.73 c_{s}\right)$. A parabolic fit (dashed red curve) agrees well with the crack profile for a distance larger than $5 \mathrm{~mm}$. (right) A closeup view $(1.0 \times 1.8 \mathrm{~mm})$ reveals a deviation, $\delta$, between the tip of the parabola and the crack tip. (b) $\delta$ and (c) the parabola curvature, $a$, as functions of $v / c_{s}$.

(CTOD), which is the clearest manifestation of the displacement field in the cracked body. LEFM predicts a parabolic CTOD:

$$
x^{\prime}=-\frac{32 \pi(1+T / E)}{\left[3 K_{I} / E \cdot \Omega_{y}(\theta=\pi, v)\right]^{2}} \cdot y^{\prime 2} \equiv-a \cdot y^{\prime 2} .
$$

We define moving frame coordinates, $(r, \theta) \quad(r=$ $\sqrt{(x-v t)^{2}+y^{2}}$ and $\left.\theta=\tan ^{-1}[y /(x-v t)]\right)$, centered at the crack tip where $\theta=0$ is the propagation direction. In Eq.(10), $K_{I}$ is the stress intensity factor, $T$ is the subleading correction known as the "T-stress" and $\Omega_{x, y}(\theta ; v)$ are universal functions [8] of $\theta$ and the crack velocity, $v$. As $T / E \ll 1$, the curvature, $a$, of the parabola provides a direct measurement of $K_{I}$. This important quantity, according to LEFM, wholly dictates the behavior of a moving crack.

To obtain $K_{I}$, we extracted the crack opening profiles from the photographs and fitted them to parabolic forms over $1-2 \mathrm{~mm}$ from the crack tip, which is within the range where LEFM might be expected to be relevant. The parabolic profiles obtained from the fitted data agreed well with CTOD profiles at significantly larger scales, indicating their robustness. For example, the fitted parabola in Fig. 1(a) is indistinguishable from the CTOD profile over $5 \mathrm{~mm}$. The crack-tip curvature is seen to be a decreasing function of the velocity [Fig. 1(c)]. Interestingly, it is well described by a linear function with its extrapolated zero intercept at a velocity close to $c_{s}$.

A closer look, however, [Fig. 1(a) right] reveals that a noticeable deviation between the fit and the crack edges occurs in the near vicinity of the tip. This deviation, $\delta$, increases rapidly with crack velocity, from $\sim 30 \mu \mathrm{m}$ at

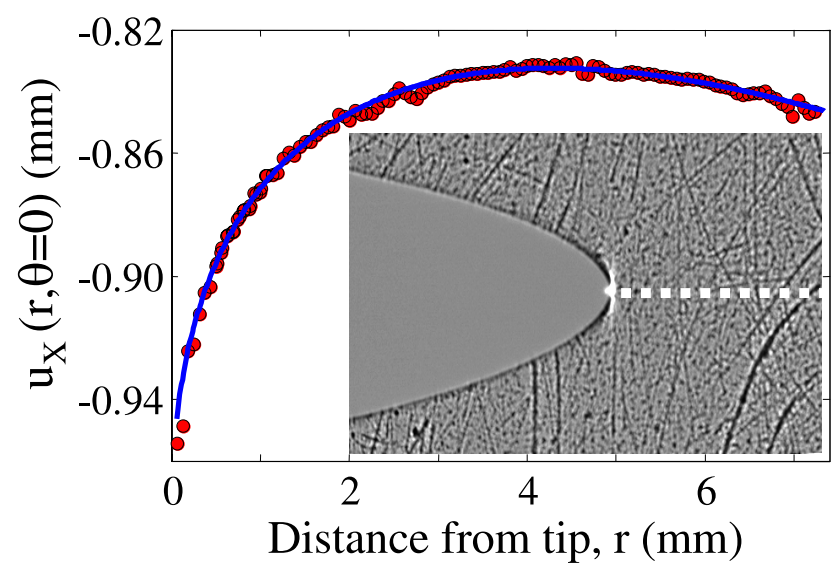

FIG. 2: (Color online) Scratch patterns (inset) imprinted on the gels are used to measure the displacement field surrounding the crack tip via particle tracking. The measured $u_{x}$ $\left(v=0.37 c_{s}\right)$ along the $\theta=0$ axis of symmetry (dashed white line) is fitted to Eq. (2). The values of $u_{x}$ are accurate up to a small additive constant $(<0.05 \mathrm{~mm})$.

low velocities $\left(\sim 0.2 c_{s}\right)$ up to $\sim 300 \mu \mathrm{m}$ at high velocities $\left(\sim 0.8 c_{s}\right)$ [Fig. 1(b)]. The near-tip region is one of considerably higher curvature than the "far-field" regions captured by the parabolic form. Attempts to force the tip of the parabola to coincide with the measured crack tip yielded terrible compatibility with the data at all scales. We are therefore led to the conclusion that the near-tip region described by $\delta$ is not described by LEFM. We will use $\delta$ to characterize the scale of this divergence. Although somewhat arbitrary, other length-scales (e.g. the distance between the crack tip and the point where the divergence begins) display similar functional behavior.

The near-tip region characterized by $\delta$ can not be explained in the framework of LEFM. A natural explanation for this region of deviation would be to associate it with the "process zone" scale, where elasticity breaks down due to extreme stresses, giving way to plastic deformations and fracture itself. LEFM avoids any treatment of this zone and regards it as a singular point under the conditions of small-scale yielding [1]. Another possible signature of the process zone may be the white region visible at the tip of the crack [Fig. 1(a) right]. In this region, the high strains induce large material deformations giving rise to lensing effects. In pictures, as this one, where the camera's focal plane is set slightly below the gel (between the gel and the light source) we observe increased light intensity in the vicinity of the crack. When the focal plane is slightly above the gel plane (between the gel and the camera), the same region becomes black. Hence the near tip region behaves as a diverging lens.

Let us now consider the displacement field $\boldsymbol{u}(r, \theta)$ around the crack tip at other $\theta$ 's. The best defined direction is along the axis of propagation directly ahead of the crack $(\theta=0)$. In Fig. 2 we present an example of the 

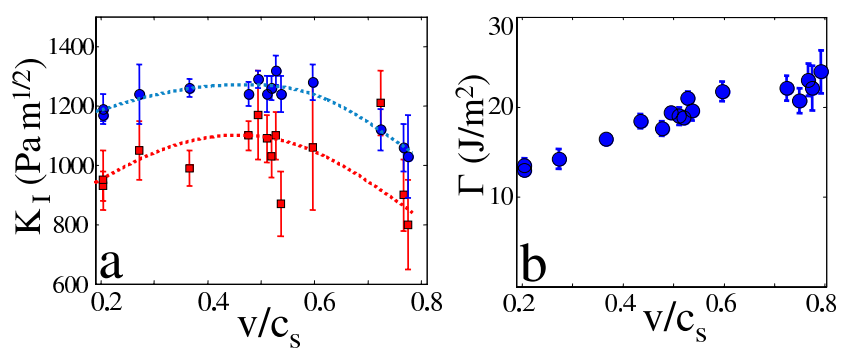

FIG. 3: (Color online) (a) The stress intensity factor, $K_{I}$, is extracted from crack opening displacement (CTOD) using Eq. (11) (circles) and from $u_{x}(r, \theta=0)$ measurements using Eq. (2) (squares). The $20 \%$ difference between the two is well beyond measurement uncertainties. Dashed lines are to guide the eye. (b) The fracture energy, $\Gamma$, calculated using $K_{I}$ (derived from the CTOD using Eqs. (1) and (3)). The weak increase of $\Gamma$ with $v$ relative to $\delta$ implies that $\delta$ is due to nonlinear elasticity, rather than dissipative processes.

measured displacements along this symmetry axis, obtained using the particle tracking method explained earlier. We first notice that the displacements are negative, since particles ahead of the crack are pulled towards it. Comparing these measurements to the predicted LEFM functional form [1]

$$
u_{x}(r, 0)=\frac{3 \Omega_{x}(0, v)}{4 \sqrt{2 \pi}} \frac{K_{I}}{E} \cdot \sqrt{r}+\frac{T}{E} \cdot r+\text { Const },
$$

we obtain excellent agreement at all measured scales, from distances well beyond $\delta(v)(7 \mathrm{~mm}$ in Fig. 2) to the closest near-tip vicinity that we are able to measure $[9]$. Eq. (2) contains the contribution of the T-stress as well, since the K-field alone is insufficient to describe the data. Thus in the region of study we have $K$-T dominance instead of the typically assumed $K$-dominance. The next order term in the $u_{x}$ expansion $\left(r^{3 / 2}\right)$ is the first to incorporate dynamic corrections due to non-steady state contributions like $\dot{v}$ and $\dot{K}_{I}[1]$. We found no evidence in our data for the existence of such contributions.

We are now in a position to compare the values of $K_{I}$ extracted from the two different methods: the displacement field along the symmetry axis (using Eq. (2)) and the CTOD measurements (using Eq. (10). We expect to find quantitative agreement between the two measurements, as LEFM predicts that $K_{I}$ is unique and has no angular dependence. Curves of $K_{I}(v)$ extracted from both measurements are presented in Fig. 3(a). Although both describe a similar functional profile: $K_{I}$ increases slowly until reaching a peak at $v \sim 0.5 c_{s}$ before decreasing again, surprisingly, the two $K_{I}(v)$ curves quantitatively differ. The systematic $20 \%$ difference in the values of $K_{I}$ is much larger than our measurement error and does not appear to be $v$-dependent. As our measured values of $T / E$ are less than a few percent, any uncertainty in their value could not explain this large deviation in $K_{I}$.

Unlike the quantitative discrepancy, the qualitative be-

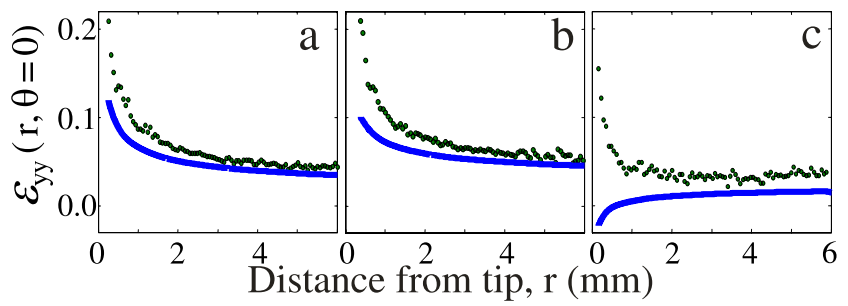

FIG. 4: (Color online) The measured strain, $\varepsilon_{y y}(r, \theta=0)$, (dots) is compared to the theoretical (LEFM) prediction (solid curve): $\left[g_{y}(\theta=0, v) \cdot K_{I} / E\right] / \sqrt{r}-0.5 T / E$ where $K_{I}$ and $T$ are taken from the $u_{x}$ fit (cf. Fig. 2) and $g_{y}$ is a known universal function [1]. The discrepancy between the two increases with the crack velocity: (a) $v=0.20 c_{s}$, (b) $v=0.53 c_{s}$, (c) $v=0.78 c_{s}$. For the higher velocity (c), LEFM predicts a negative strain (compression) ahead of the crack tip.

havior of $K_{I}$ is easily understood. According to LEFM, $K_{I}$ factorizes into a universal dynamic component, which depends solely on the velocity and a geometric component, which depends on the loading and dimensions of the stressed object. While the first is a decreasing function of $v$, the latter increases with $v$ through the loading. The non-monotonicity of $K_{I}(v)$ is due to the competition between these two components.

Using $K_{I}(v)$, we can now compute the material's fracture energy, $\Gamma[1]$

$$
\Gamma(v)=K_{I}^{2}(v) A_{I}(v) / E
$$

where $A_{I}(v)$ is a known [1] function of $v$. Using the values of $K_{I}(v)$ obtained from the CTOD, we observe [Fig. 3(b)] that $\Gamma$ is a slowly monotonically increasing function of $v$ whose value is approximately $20 \mathrm{~J} / \mathrm{m}^{2}$. Similar values have been reported for other gels [10]. $\Gamma$ is a measure of the dissipation involved in crack propagation. It cannot, in general, be estimated from first principles, but is a material property which must be measured.

We are now in a position to understand the nature of the processes that govern the deviations $\delta$ from the parabolic CTOD. In principle, $\delta$ can either be the scale of dissipative processes (i.e. the process zone) or it may be associated with nonlinear elastic processes. If the former were correct, one would expect $\Gamma(v)$ and $\delta(v)$ to have a similar functional dependence. Comparison over the velocity range of $0.4-0.8 c_{s}$ reveals only a modest $\sim 30 \%$ increase in $\Gamma$, while $\delta$ increases by over $\sim 400 \%$. This large contrast indicates that nonlinear (non-dissipative) elastic processes are dominant in the near-tip region at scales that are significantly larger than dissipative ones.

The large discrepancy between the two values of $K_{I}$ derived from different components of the same displacement field, motivated us to examine also the deformation in the y-direction at $\theta=0$. Since for Mode I symmetry, $u_{y}(r, 0)=0$, we instead use the strain $\varepsilon_{y y}(r, \theta=0)=$ $\partial_{y} u_{y}(r, 0)$. In Fig. 4 we compare $\varepsilon_{y y}(r, 0)$ at three different velocities, from $20 \%$ to $\sim 80 \%$ of $c_{s}$, to the LEFM 
predictions [11]. At all velocities we measure strains of $\sim 0.2-0.3$, before encountering measurement limitations [9] when too close $(\sim 200 \mu \mathrm{m})$ to the tip. LEFM predictions for $\varepsilon_{y y}(r, 0)$ were calculated using the $K_{I}$ and $\mathrm{T}$ stress values obtained by fitting the $u_{x}(r, 0)$ displacement components at the same velocities, cf. Fig. 2.

In stark contrast to the excellent fits obtained for $u_{x}$, the predicted values of $\varepsilon_{y y}$ deviate significantly from the measured ones. At the lower velocities, the measured strain increases notably more rapidly than the $r^{-1 / 2}$ dependence predicted by LEFM [Fig. 4(a-b)], and attempts to fit the strains using LEFM fail. At higher velocities [Fig. 4(c)] the deviations between measured and expected values are even more dramatic.

For $v>0.73 c_{s}$ (for incompressible materials) LEFM predicts a monotonically decreasing strain which reaches negative values (compressive strains) as one approaches the crack tip. On the other hand, our measurements show the strain to be a positive, monotonically increasing function that reaches near-tip values that are similar to those measured at lower velocities. The measurements make more intuitive sense than the LEFM predictions tensile fracture occurs under extension.

The experiments described here present high resolution measurements of the displacement field surrounding dynamic cracks. We show that the canonic theory of fracture, LEFM, fails to provide a consistent description of the experimental data, apparently as a result of elastic nonlinearities [12]. This, by itself, is neither surprising nor contradicts LEFM, as these effects may be conceptually incorporated into one of its key assumptions, the small-scale yielding condition [1].

On the other hand, as fracture occurs precisely at the smallest scales, a description of this near-tip region is important. Above, we presented detailed measurements within the nonlinear region in this type of material. In addition, we demonstrated that at high $v$, LEFM qualitatively fails to describe the tensile extensions measured ahead of the crack tip cf. Fig. 4(c). This underlines the necessity for a more complete theoretical understanding of essential nonlinear effects.

Are these observations unique to elastomers or are they more generally valid? In the accompanying Letter [8] we develop a weakly nonlinear theory of the dynamic fracture of a single crack that resolves all of the discrepancies discussed above. Such nonlinearities are as universal as linear elasticity and must be experienced by any material undergoing fracture. Therefore, we expect these results to be generally applicable to any brittle material.

The key features of brittle fracture in the gels considered here are identical to those observed in other brittle amorphous materials, like glassy polymers or structural glasses [6]. This work, however, indicates that LEFM is unable to describe these features in gels, since the neartip fields are nonlinearly elastic at scales encompassing the origin of these effects. Moreover, the near-tip dissipative processes in gels, brittle plastics and glasses are as different as their micro-structure. This leads us to conclude that the nonlinear elastic region that bridges the gap between LEFM and the process zone must play a critical role in governing the fracture process. Thus, understanding the dynamics within this region may be the key to unlocking a plethora of open questions that are related to the breakdown of single straight cracks. These include questions of stability [2] (e.g. micro-branching and crack oscillations), crack path selection [2], and 3D nonlinear focussing (e.g. crack front inertia, front wave nonlinear structure, and the formation of directed chains of micro-branches [6]).

Acknowledgements This research was supported by grant 57/07 of the Israel Science Foundation. E. B. acknowledges support from the Horowitz Center for Complexity Science and the Lady Davis Trust.

[1] L. B. Freund, Dynamic Fracture Mechanics (Cambridge University Press, Cambridge, 1990).

[2] J. Fineberg and M. Marder, Phys. Rep. 313, 1 (1999).

[3] A. Livne, O. Ben-David and J. Fineberg, Phys. Rev. Lett. 98, 124301 (2007).

[4] For a review of near-tip measurements see: K. RaviChandar, Dynamic Fracture (Elsevier, Amsterdam, 2004).

[5] F. Célarié et al., Phys. Rev. Lett. 90, 075504 (2003).

[6] A. Livne, G. Cohen and J. Fineberg, Phys. Rev. Lett. 94, 224301 (2005).

[7] Collimated light (LED source) oriented normal to the gel faces was deflected by the scratches imprinted on the gel surface. Optimal contrast was obtained by setting the focal plane of the camera slightly below that of the gel.

[8] E. Bouchbinder, A. Livne and J. Fineberg, following Letter, ?? (2008).

[9] The measured tip location is used in the fitted function. Note that we are unable to measure displacements at points closer than $\sim 200 \mu \mathrm{m}$ from the tip, due to the extreme strains and lensing effects that dominate there.

[10] Y. Tanaka, K. Fukao and Y. Miyamoto, Eur. Phys. J. E. 3, 395 (2000); T. Baumberger, C. Caroli and D. Martina, Nature Mat. 5, 552 (2006).

[11] We evaluated $\varepsilon_{y y}(r, \theta=0)$ in the following manner: for every distance $r$ from the crack tip along $\theta=0$, $\varepsilon_{y y}(r, \theta=0)$ was obtained from the linear term of a 3 rd order polynomial fit of $u_{y}$ along $y$ (without the quadratic term), where $\leq 16$ measured values of $u_{y}$ above and below the symmetry axis were used.

[12] M. J. Buehler, F. F. Abraham and H. Gao, Nature 426, 141 (2003); M. J. Buehler and H. Gao, Nature 439, 307 (2006). 